\title{
Genetically Engineered Mouse Models of Pancreatic Cancer
}

\author{
C. Benedikt Westphalen ${ }^{1,3}$ and Kenneth P. Olive ${ }^{1,2,3,{ }^{*}}$ \\ ${ }^{1}$ Department of Medicine, Division of Digestive and Liver Diseases, Columbia University Medical \\ Center, New York, NY 10032, USA \\ ${ }^{2}$ Department of Pathology, Columbia University Medical Center, New York, NY 10032, USA \\ ${ }^{3}$ Herbert Irving Comprehensive Cancer Center, Columbia University Medical Center, New York, \\ NY 10032, USA
}

\begin{abstract}
Pancreatic ductal adenocarcinoma is the $10^{\text {th }}$ most common cancer and the $4^{\text {th }}$ leading cause of cancer-related death in the United States. Despite great effort, the prognosis for patients with this disease remains dismal with, a 5-year survival rate of just 4-6\%. While several important advances have improved our understanding of the underlying biology of pancreatic cancer, this knowledge has not translated into novel therapeutic approaches and effective systemic or targeted therapies. Pancreatic cancer is one of the malignancies most difficult to treat, with remarkable intrinsic resistance to both standard and targeted chemotherapy as well as ionizing radiation. Surgical intervention remains the only potentially curative approach. However, most patients present with inoperable and/or metastatic disease and are therefore excluded from surgery. Accordingly, new therapeutic options are desperately needed. In vivo models to study innovative and alternative treatment approaches are of major importance. A variety of genetically engineered mouse models of pancreatic cancer have been developed over the last decade. However, these models display different characteristics and not all of them are suited for preclinical studies. In this review we aim to review the mouse models currently available, their experimental use, their clinical relevance and limitations as well as future directions.
\end{abstract}

\section{Keywords}

Pancreatic Cancer; genetically engineered mouse models; transgenic; preclinical testing

\section{Introduction}

In 2012, an estimated 43,920 people will be diagnosed with pancreatic ductal adenocarcinoma (PDAC) in the United States, and 33,790 people will succumb to the disease. Seventy-five percent of patients die within one year of their diagnosis and the fiveyear survival rate of $4-6 \%$ has remained unchanged for the last four decades. ${ }^{1,2}$ In recent years, significant progress has been made in understanding the biology of pancreatic cancer.

\footnotetext{
*Address correspondence to: Departments of Medicine and Pathology Herbert Irving Comprehensive Cancer Center Columbia University Medical Center 1130 Saint Nicholas Avenue, ICRC 217A New York, NY 10032, USA kenolive@ columbia.edu Phone: (212) 212-851-4678

Conflict of interest: - none relevant.

This is a PDF file of an unedited manuscript that has been accepted for publication. As a service to our customers we are providing this early version of the manuscript. The manuscript will undergo copyediting, typesetting, and review of the resulting proof before it is published in its final citable form. Please note that during the production process errors may be discovered which could affect the content, and all legal disclaimers that apply to the journal pertain.
} 
In contrast to many epithelial malignancies, PDAC is distinguished by four genes that are altered in a very high fraction of patients: the K-ras proto-oncogene is mutationally activated in $>90 \%$ of cases, while the tumor suppressors CDKN2A, p53, and DPC4/SMAD4 are altered in $>95 \%, 50-75 \%$, and $55 \%$ of cases, respectively. Several types of precursor lesions have been identified that can lead to PDAC, including pancreatic intraepithelial neoplasia (PanINs), intraductal papillary mucinous neoplasia (IPMNs) and mucinous cystic neoplasia (MCNs). PanINs in particular are characterized by a step-wise acquisition of mutations from low-grade dysplasia (PanIN 1-2) to carcinoma in situ (PanIN 3). ${ }^{3,4}$ Given the frequency of K-ras mutations in PDAC and their presence in the earliest PanIn lesions, activation of oncogenic K-ras signaling has been proposed to be the primary initiating event in pancreatic carcinogenesis.

Layered on top of this framework of four high-penetrance genetic alterations is a stunning background of low-penetrance mutations that are now known to occur, in some combination, in nearly all pancreatic tumors. In a milestone Science paper published in 2008, Jones et al used high throughput sequencing, gene expression, and copy number analysis techniques to define the genetic landscape of ductal pancreatic cancers. While no new high penetrance genes were identified, this study was important for its detection of over 1000 total somatic mutations, with each tumor averaging over 60 mutations attributable to 12 core-signaling pathways in pancreatic ductal adenocarcinoma ${ }^{3}$. This complexity highlights the need for carefully tailored approaches to the treatment of individual patients, while also emphasizing the importance of tackling the core pathways that define this disease.

Another hallmark of pancreatic ductal adenocarcinoma is a profound fibrous inflammatory reaction termed desmoplasia. This stromal component of the tumor can comprise up to $90 \%$ of the mass, and includes fibroblasts, leukocytes, endothelial cells, and extracellular matrix proteins. Initially regarded as the body's response against the developing tumor, it is now widely accepted that desmoplasia is actively driven by signals from epithelial cells and plays a role in both tumor development, progression, and chemotherapeutic resistance. ${ }^{5}$

While the recent development in our understanding of the underlying biology of PDAC has not yet led to improved patient care, this knowledge has been the foundation for the development of new genetically engineered mouse models that have provided both further basic insights into the disease as well as several novel therapeutic approaches that are now being translated into the clinic. The goal of this review is to provide an overview of the common transgenic mouse models of pancreatic cancer, to discuss their specific use and limitations in basic and translational research, and to highlight unmet needs in the area of mouse models of pancreatic ductal adenocarcinoma.

\section{Modeling the biology of pancreatic cancer in mice}

While different genetic and chemical approaches to generate pancreatic cancer in mice and other rodents date back to the early $1980 \mathrm{~s}^{6-8}$, it was the generation of the K-ras ${ }^{\text {LSL.G12D }}$ mouse $^{9,10}$ in 2001 that allowed tissue-specific expression of mutant K-ras under physiological control from the endogenous mouse locus. The backbone of this mouse is a targeting construct containing a transcriptional and translational silencing cassette (STOP) flanked by two loxP sites, residing upstream of exon 1 in the K-ras gene. This "LSL cassette" prevents expression of the allele, which also contains an additional G-A transition that yields a glycine to aspartic acid substitution in codon 12, the most commonly mutated residue K-ras in human PDAC. This mutation alters the intrinsic GTPase activity of Kras, ${ }^{11-13}$ as well as its affinity for GAPs, locking the protein into the inactive, GTP-bound configuration. ${ }^{14}$ Expression of the bacterial recombinase cre results in removal of the LSL 
cassette, expression of mutant K-ras ${ }^{\mathrm{G} 12 \mathrm{D}}$ from its endogenous locus, and thereby activation of the Ras pathway.

In 2003, the groups of David Tuveson and Ronald DePinho each took advantage of the Kras ${ }^{\text {LSL.G12D }}$ mouse to generate new models of PDAC that recapitulate the step-wise development of cancer seen in humans. ${ }^{15,16}$ During development, all pancreatic cells are derived from pan-pancreatic progenitors labeled by the transcription factors PDX and Ptf1ap48 (p48). ${ }^{17}$ By crossing PDX-Cre ${ }^{18,} 19$ or $\mathrm{p} 48$-Cre ${ }^{20}$ mice to K-ras ${ }^{\text {LSL.G12D }}$ mice, the authors targeted expression of K-ras ${ }^{G 12 D}$ specifically to the pancreas. Surprisingly, the mice were born with normal pancreatic histology and architecture. An increased pancreatic mass at birth was the only difference between double transgenic mice and controls. However, by 8 weeks of age, the mice began to develop early PanIn (mPanIN 1A/B) lesions that slowly increased in both number and grade over the next 2 years. A subset of these mice developed pancreatic ductal adenocarcinoma, with a median overall survival of 14 months. ${ }^{21}$ It is worth noting that the $\underline{\mathrm{K}}-\mathrm{ras}^{\mathrm{LSL} . \mathrm{G} 12 \mathrm{D} /+}$; Pdx$\underline{\mathrm{Cre}}$ model (now referred to as the $\mathrm{KC}$ model) developed a significant background tumor spectrum due to leakiness of the PdxCre allele in other tissues, most notably gastric papillomas and keratoacanthomas of the anal and oral mucosae. These background tumors are less common when the more-specific p48-Cre allele is used.

The KC model provided proof that K-ras mutations are sufficient to initiate pancreatic cancer formation in mice ${ }^{18}$. In contrast, targeted conditional mutations in cdkn2 $\mathrm{A}^{16}, \mathrm{p}^{12} 3^{22}$, or smad4 $4^{23}$ did not lead to tumor or PanIN development in the context of PdxCre expression. Nonetheless, the long latency and infrequent progression to invasive cancer limited the utility of this KC model for preclinical applications. Thus, additional targeted mutations were crossed into the $\mathrm{KC}$ model. Aguirre et al. found that homozygous deletion of cdkn2a (which encodes the p16 $6^{\text {Ink4a }}$ and p19Arf tumor suppressors) in the context of K-ras mutation in the pancreas led to the rapid development of highly aggressive and metastatic ductal adenocarcinoma, causing $100 \%$ lethality at eleven weeks. ${ }^{16}$ These mice displayed clinical features of pancreatic cancer such as biliary obstruction, weight loss and hemorrhagic ascites. Interestingly, the authors also observed the development of pancreatic tumors with sarcomatoid histology in mice with homozygous deletion of cdkn2a (Kras $^{\text {LSL.G12D/+; }}{ }^{\text {cdkn2a }}{ }^{\text {lox/lox}} ;$ PdxCre).${ }^{16}$

Similarly, the conditional expression of the tumor-associated $\mathrm{p} 53^{\mathrm{R} 172 \mathrm{H}}$ mutation also accelerated K-ras ${ }^{\mathrm{G} 12 \mathrm{D}}$ pancreatic tumorigenesis ${ }^{21}$. While, born with histologically normal pancreata, $\underline{\mathrm{K}}-\mathrm{ras}^{\mathrm{LSL} . G 12 \mathrm{D} /+} ;$ p53 ${ }^{\mathrm{R} 172 \mathrm{H} /+} ;$ Pdx Cre mice (now referred to as KPC mice) developed PanIN lesions on an accelerated schedule, and died of PDAC with a median survival of 5.5 months. Metastases were observed $\sim 80 \%$ of the animals, at the same sites seen in human PDAC patients (liver, lung, and peritoneum). Tumors arising in this model were found to have many of the immunohistochemical markers associated with human disease, and bore evidence of widespread genomic alterations, a feature that was previously lacking in most genetically engineered mouse models. Sarcomatoid pancreatic cancers were also occasionally observed in this model, though less frequently than in mice with homozygous deletion of cdkn2a. ${ }^{22}$ Interestingly, subsequent reports of mice bearing a

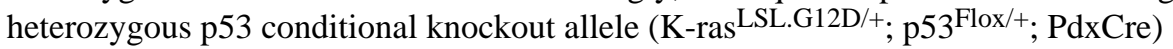
described reduced invasiveness and decreased metastasis, and provided support for a "gain of function" effect conferred by the point-mutant p53 allele. ${ }^{24,25}$

Three models were reported that addressed the role of TGF- $\beta$ pathway alterations in the context of K-ras ${ }^{\mathrm{G} 12 \mathrm{D}}$ expression. Ijichi and colleagues crossed the K-ras ${ }^{\text {LSL.G12D }}$ model with a knockout of the TGF- $\beta 2$-receptor (Tgfbr2), resulting in rapid onset of PanIn formation and progression to PDAC with a median survival of eight weeks. Interestingly, 
the authors reported the absence of histologies other than ductal adenocarcinoma in these mice. While metastatic disease was difficult to assess due to their short overall survival, in rare cases older mice exhibited metastatic spread to typical sites. ${ }^{26}$ Interestingly, pancreasspecific loss of SMAD4 in the context of K-ras ${ }^{\mathrm{G} 12 \mathrm{D}}$ expression, a common combination in human PDAC, ${ }^{1}$ did not recapitulate the knockout of Tgfbr2. Rather, these animals developed cystic tumors that progressed to invasive PDAC. The two groups that reported these mice respectively characterized their histology as gastric-type IPMNs (based on mucin expression) ${ }^{27}$ or MCNs (based on the presence of progesterone-receptor positive "ovarian stroma"). ${ }^{28}$

While the clinical and histological appearance of the genetically engineered mouse models (GEMMs) described above closely resembles the human condition, the relevance of these models to human cancer is often questioned. Recently, Collisson et al used next generation transcriptional profiling of primary tumor samples and established cell lines to define three different subtypes of pancreatic ductal adenocarcinoma. ${ }^{29}$ Importantly, the authors found the same signatures in cell lines derived from different GEMMs of pancreatic cancer, validating these models in an unbiased way. These GEMMs now provide powerful tools to investigate the basic biology of pancreatic cancer and the role of specific genes in pancreatic cancer initiation, progression and maintenance. Furthermore numerous groups have begun to employ these models in a preclinical setting for the purpose of advancing translation of novel therapies. The following sections demonstrate how GEM models of PDAC have been used to address both basic and translational problems.

\section{Studying gene function in GEM models of PDAC}

Much of the progress made in recent years in defining the role of specific genes in tumorigenesis has occurred due to the use of GEMMs. Table 1 provides a list of genetic combinations that have been reported to develop PDAC (or premalignancies) to date. While the earliest efforts focused largely on the core pathways described above, recent efforts have focused on understanding the subtleties of K-ras signaling, and on the search for critical pathways necessary for tumor maintenance that could be targeted therapeutically.

Fully understanding the function of mutant K-ras in PDAC remains a critical goal for the field. The K-ras ${ }^{\text {LSL.G12D }}$ strain has been extensively utilized as the genetic backbone of studies into numerous aspects of the biology of pancreatic cancer. However, following Cre expression, this allele remains constitutively activated, limiting its utility for studying the role of mutant K-ras in tumor maintenance. Recently, two groups independently and elegantly addressed the issue of tumor maintenance using a tetracycline-inducible allele of mutant K-ras. After initiating K-ras ${ }^{\mathrm{G} 12 \mathrm{D}}$-driven tumorigenesis by treating the mice with doxycycline, both groups found that withdrawal of the drug (and therefore cessation of mutant K-ras expression) led to the regression of established pancreatic tumors. The acute loss of mutant K-ras quickly resulted in profound alterations in tumor cell metabolism and dramatic reorganization and involution of the desmoplastic stroma. ${ }^{30,31}$

While these data emphatically confirm the premier role of K-ras in the biology of pancreatic cancer, efforts to directly target K-ras with therapeutics have not been successful to date. ${ }^{32}$ Therefore, Ras effector pathways such as Raf-MEK-ERK and PI3K-AKThave been investigated as potential surrogates that are more suitable for therapeutic intervention. While genetically engineered mouse models specifically investigating the Raf-MAPK in pancreatic cancer have not been reported to date, there is growing evidence for a complex role of PI3K signaling in pancreatic cancer. For example, loss of PTEN in the pancreas results in metaplasia and expansion of centroacinar cells, with rare progression to malignancy. 33 Combining PTEN deletion with K-ras mutation leads to dramatic acceleration of tumorigenesis with a fully penetrant, highly aggressive phenotype. ${ }^{34-36}$ Conversely, deletion 
of the Ras effector Rac from K-ras ${ }^{\mathrm{LSL} . G 12 \mathrm{D} /+}$ p48-Cre mice resulted in reduced PanIN formation and extended overall survival. ${ }^{37}$

The function of a number of other individual genes has been studied in the context of Kras ${ }^{\mathrm{G} 12 \mathrm{D}}$ induced tumorigenesis by crossing into the $\mathrm{KC}$ model (or a variant thereof). This reverse genetics approach, while informative, is typically a slow process that requires foreknowledge of which genes are important to study. As an alternative approach, the laboratories of David Tuveson and Nancy Jenkins/Neil Copeland recently reported the use of transposon-mediated mutagenesis to carry out forward mutagenesis screens for genes that promote K-ras ${ }^{\text {G12D }}$ initiated pancreatic tumors. ${ }^{38,39}$ Both groups utilized the Sleeping Beauty transposon system, ${ }^{40}$ a genetic tool that allows mobilization of transposable elements that can promote or interfere with gene expression following their (roughly) random integration within the genome. By crossing to $\mathrm{KC}^{18}$ mice and analyzing the location, orientation, and frequency of transposon insertions in the resulting tumors, the two groups identified a large number of candidate genes that could promote tumor progression. Importantly, well-known pathways such as TGF- $\beta$ and p16/CDKN2A were found to be affected, and there was a significant degree of overlap between the genes reported by both groups. Both teams identified genes involved in chromatin remodeling, including Usp9X, ${ }^{39}$ as playing an important and previously unappreciated role in the pathogenesis of PDAC.

\section{The Cell of Origin in Pancreatic Cancer}

The cell of origin in pancreatic cancer has been a point of considerable debate. ${ }^{41}$ While the histological appearance of pancreatic cancer suggests a ductal cell of origin, careful genetic studies have shown that a variety of different pancreatic cell types may undergo malignant transformation under different experimental conditions ${ }^{41}$. Accordingly, a range of different cell types have been proposed as putative pancreatic progenitor and cancer initiating cells, including preexisting acinar cells, preexisting $\beta$-cells, pancreatic ductal cells, and cells expressing the mesenchymal marker nestin ${ }^{42}$, 43 . Classical mouse models of pancreatic cancer depend on the expression of oncogenes and loss of tumor suppressors in panpancreatic progenitor cells during embryonic development, and are therefore unsuitable for investigations into the cell of origin. The first evidence for a non-ductal origin of pancreatic

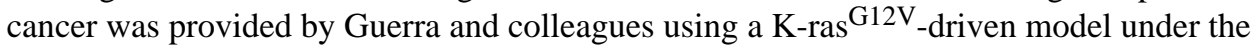
control of a tetracycline-regulatable elastase promoter, which allowed for inducible expression of mutant K-ras in the acinar cells. Induction of mutant K-ras shortly before birth led to the development of acinar to ductal metaplasia, the formation of mPanINs, and progression towards invasive PDAC. Interestingly, induction of K-ras ${ }^{\mathrm{G} 12 \mathrm{~V}}$ in adult mice failed to induce malignant transformation. Additional pancreatic injury in the form of caerulein administration restored the ability of elastase-expressing cells to initiate pancreatic cancer in adult mice. Subsequent studies by Gidekel Friedlander et $\mathrm{a}^{44}$ and Morris et al ${ }^{45}$ extended our understanding of the plasticity of differentiated pancreatic cells in the setting of K-ras mutation and additional tissue injury in the adult. Most recently, studies focusing on the malignant potential of nestin-positive cells or Sox9 positive cells have focused attention on these rare cell types within the adult pancreas. ${ }^{46-49}$ However, all of these studies have been plagued by the technical limitations of current transgenic and cre-lox technologies; the low-level expression of Cre in rare (and therefore hard-to-detect) cells of the adult pancreas could lead to mistaken interpretations. For example, low-level leakiness of several transgenic cre lines has been found in "centroacinar cells", a putative progenitor cell type found in the adult pancreas. These poorly characterized Hes1 expressing cells lie at the junction between acinar cells and the terminal ductal epithelium, and some studies have presented evidence for malignant transformation from the centroacinar compartment. ${ }^{33,5051}$ However, specific expression of mutant K-ras in adult centroacinar cells using inducible Cre-lox technology has not been reported to date. Resident tissue stem cells are attractive 
candidates for cancer initiating cells in the adult. While bone fide stem cells have been identified through Cre-mediated lineage tracing for a number of tissues with rapid turnover (such as the skin, intestine, etc.), pancreatic stem cells have not been as clearly defined. ${ }^{52,53}$ Taken together, GEM models have suggested many candidates for the cell of origin of PDAC, but have failed to provide a conclusive determination.

\section{The role of stromal desmoplasia}

Unlike cell lines, organotypic cell cultures, or even xenograft systems, GEM models can fully recapitulate the complex relationship of neoplastic epithelial cells with their microenvironment. Desmoplastic stroma production is driven through a variety of cancer cell-derived pathways, including Tgf- $\beta$, as well as epidermal, hepatocyte, fibroblast and insulin-like growth factors. ${ }^{5}$ These pathways and others engage with the various cell types that comprise the stroma, including leukocytes, endothelial cells, neuronal cells, and a variety of different types fibroblasts. While some of these components have previously been studied using transplantation models such as xenografts, the stroma in such tumors does not closely reflect the biology or histopathology of human PDAC. In contrast, the GEMMs described above have enabled for the first time the study of a physiologically accurate autochthonous tumor in an immune-competent setting. It has long been appreciated from histological studies of human PDAC that neoplastic epithelial cells are minority cell types within the tumor mass. Careful flow cytometry analysis of fresh PDACs from the KPC model revealed that nearly half the cells in KPC tumors are leukocytes, with a particularly large number of myeloid derived suppressor cells. ${ }^{54}$ These cells help to mediate the profoundly immunosuppressive microenvironment that enables ductal pancreatic tumors to escape the notice of the immune system. One of the underlying molecular signals that drives this process was recently illuminated in a pair of seminal papers from the groups of Dafna Bar-Sagi ${ }^{55}$ and Robert Vonderheide. ${ }^{56}$ Using complementary approaches, both teams found that activation of K-ras ${ }^{\mathrm{G} 12 \mathrm{D}}$ expression results in upregulation of the cytokine GM-CSF, which signals to immature myeloid progenitor cells and promotes their activation into myeloid-derived suppressor cells (MDSCs). Notably, anti-GM-CSF antibodies were effective at blocking the accumulation of MDSCs in the tumor stroma, thereby enabling $\mathrm{CD}^{+} \mathrm{T}$-cells to inhibit tumor growth. Both studies relied on GEM models of pancreatic cancer or cells derived from them.

Another aspect of the stroma that came to be appreciated through work in GEMMs is the role of the tumor vasculature. It has become canon that angiogenesis is a mandatory process during tumor progression. However, work in the KPC model brought attention to PDAC as an intriguing counter example. ${ }^{57}$ In studying the nature of primary chemoresistance, our group used both in vivo imaging studies and ex vivo microscopy and pharmacology techniques to demonstrate that pancreatic tumors are poorly perfused, due to a paucity of functional blood vessels. Indeed, radiologists have long diagnosed PDAC based on the observation of a "hypo-enhancing mass" in the pancreas. The fact that numerous different contrast agents are unable to access the tumor should be considered in trying to understand why therapeutic agents, such as genotoxic chemotherapies, are so rarely effective in this disease. This effect appears to be mediated by other components of the desmoplastic stroma.

Another important mediator of desmoplasia is the sonic hedgehog (SHh) pathway. During development, this pathway coordinates the growth of epithelial and stromal cells in numerous organ systems. In PDAC, this system has been co-opted to promote stromal desmoplasia. Overexpression and secretion of the SHh ligand is a common feature in neoplastic epithelial cells of PDAC. ${ }^{58}$ However, downstream signaling is restricted to nearby stromal cells, forming a paracrine axis that drives the proliferation of stromal cells. ${ }^{59-62}$ The delineation of this pathway has led to the preclinical and clinical investigation of inhibitors of the hedgehog pathway in PDAC (see below). 
GEM models have also been useful in understanding the role of another developmental morphogen system in PDAC, the Notch pathway. Notch signaling plays is major role in the development of the normal pancreas, ${ }^{63}$ but is largely inactive in the adult pancreas; expression of the downstream target Hes 1 is restricted solely to centroacinar cells, a putative adult stem cell. ${ }^{64}$ During tumorigenesis, the pathway is altered and upregulated. There are many different Notch ligands and receptors, some of which are expressed in the epithelium and others in various stromal cell types. ${ }^{65}$ Thus the notch pathway may mediate multidirectional communication between various cell types within the tumor.

By providing ready access to intact ductal pancreatic tumors that harbor all of the cell types observed in human PDAC, GEM models have made it possible to study the complex signals that mediate cellular interactions within the stroma. Undoubtedly, other paracrine signals exist in PDAC that will fill in our understanding of the role of the pancreatic tumor stroma. However, the pathways identified to date provide intriguing targets for therapeutic intervention, some of which are already beginning to be explored both preclinically and clinically.

\section{The use of GEM models in preclinical studies}

The development of GEM models bearing a strong histopathological resemblance to human PDA opened the door to translational studies, with two goals: a) to determine which drugs should progress (or not) to clinical trials in humans, and b) to provide mechanistic insight into the response (or resistance) of pancreatic tumors to treatment. One initial barrier was the need for a facile technique for monitoring tumor growth in GEMMs. The broad survival curve of the KPC model and others with the most accurate histopathology precluded the use of age-based enrollment studies, since most of these animals will not harbor a tumor at any given time point. Our group has utilized high resolution 3D ultrasound as a means to monitor and quantify tumor volumes in mice with in situ pancreatic tumors. ${ }^{57,66}$ By using image-based enrollment criteria, one can perform intervention survival studies akin to those performed in clinical trials that measure the overall survival of patients from the time of enrollment.

A critical question at the outset was whether GEM models of PDA would better predict drug efficacy than existing xenograft models. This question was addressed in a side-by-side comparison of the response of several types of models to treatment with gemcitabine, the national standard-of-care therapy at the time. Reassuringly, the KPC model was found to be resistant to treatment with gemcitabine monotherapy, with only $12 \%$ of tumors demonstrating even a transient decrease in tumor volume. In contrast, human xenografts as well as mouse syngeneic models (i.e, tumor cells from the KPC model implanted orthotopically into littermate mice with an intact immune system) were both found to have a significant cytostatic response to gemcitabine treatment. ${ }^{57}$ The response of a related model (K-ras ${ }^{\mathrm{LSL} . G 12 \mathrm{D} /+}$; Ink4/Arf ${ }^{\mathrm{F} / \mathrm{F}}$; PdxCre) was shown to accurately recapitulate that of human patients treated with either inhibitors of EGFR or VEGF. ${ }^{67}$

The delineation of paracrine signaling axes in PDAC has prompted the investigation of several drugs that target these pathways. IPI-926, an inhibitor of the Hedgehog pathway effector Smoothened, was evaluated in the KPC model. Alone, the agent had no effect on tumor growth, but did result in a depletion of stroma from the tumor. When combined with gemcitabine treatment, IPI-926 improved the delivery of this and other chemotherapeutic agents, prolonged the overall survival of the animals, and decreased the incidence of liver metastases. However the translation of Smoothened inhibitors as a therapy for PDAC has met with some problems in early clinical trials. A thorough analysis of these data will be crucial for our understanding of how GEMMs can best be used for preclinical studies, and how to best translate the preclinical findings of GEMMS into clinical trials. 
Several other approaches have been taken to targeting the stroma of ductal pancreatic tumors. Cook et al explored the use of MRK003 (Merck), a gamma-secretase inhibitor, as a means to inhibit Notch pathway signaling. Gamma secretase is necessary for proteolytic processing of the Notch receptor during pathway activation. Administration of MRK003 to KPC mice effectively inhibited Notch signaling and, in combination with gemcitabine, lead to a significant improvement of overall survival of KPC mice. Importantly, the authors were able to identify the underlying mechanism of action as being mediated by the effect of Notch inhibition on the tumor vasculature, highlighting one of the principle strengths GEMMs in preparing for clinical trials.

Two groups decided to use a modified hyaluronidase (PEGPH20, Halozyme) to target the extracellular matrix of pancreatic tumors directly in the KPC model. ${ }^{68,69}$ This agent, which is now being evaluated in a Phase $1 \mathrm{~b} / 2$ clinical trial in combination with gemcitabine, quickly reduces the interstitial fluid pressure of pancreatic tumors and alters the structure of the tumor vasculature, thereby facilitating the delivery of gemcitabine. KPC mice treated with PEGPH20 plus gemcitabine lived about twice as long as those treated with gemcitabine plus vehicle.

Another way that GEM models can be utilized is to clarify the mechanistic basis of drug activity. Frese et al combine nanoparticle albumin-bound (nab)-paclitaxel (Abraxane, Celgene) and gemcitabine. Earlier studies had suggested that nab-paclitaxel, being sequestered in stroma rich cancers through binding to secreted protein, being acidic and rich in cysteine (SPARC), leads to stromal breakdown and improved response to therapy ${ }^{70,71}$. These earlier studies led to an investigator-initiated Phase II clinical trial in metastatic PDAC that demonstrated favorable effects on overall survival ${ }^{71}$. While the argument for a stromal effect of nab-paclitaxel was quite compelling, using GEMMs, Frese et al elegantly demonstrated that nab-paclitaxel improved overall survival by altering the metabolism of gemcitabine rather than improving drug delivery through decreased stromal density. ${ }^{70}$

Finally, GEM models of PDA have begun to be utilized in the evaluation of novel immunotherapies. In one of the first examples of a co-clinical trial using GEM models, Vonderheide and colleagues performed a Phase 1 study of the CD40 agonist CP-870-893 (Pfizer) in combination with gemcitabine in patients with metastatic PDAC. ${ }^{72}$ The preclinical rationale for this trial was based on data from xenograft models and was premised on the idea that activation of CD40 would reverse the immune suppression of Tcells, enabling them to attack the tumor. Although a number of patients exhibited partial responses to treatment, analysis of clinical samples did not support a T-cell mediated mechanism. In response, the authors revisited the original premise using the KPC model in order to better understand the underlying mechanism. Responses to treatment with CP-870-893 plus gemcitabine were also observed in the KPC model, but were dependent on the activation of tumoricidal macrophages rather than T-cells. Subsequent re-evaluation of the clinical samples was consistent with this new mechanism, and Phase 2 studies are now being planned.

The preclinical studies presented here are summarized in Table 2. Notably, all of these studies have been reported in the past three years, bearing witness to the explosion of preclinical research using GEM models of PDAC. Already, these studies have helped guide the clinical development of novel therapeutics and also provided fundamental insights into the molecular biology of pancreatic tumors as they respond to treatment. We anticipate many more preclinical efforts in the future, as well as additional co-clinical trials that make use of mouse and human data side-by-side, helping to build our understanding of novel agents and facilitate their clinical development. 


\section{Limitations of Current GEMMs and Future Directions}

The last decade has seen tremendous progress in the development and experimental interrogation of genetically engineered mouse models of pancreatic cancer leading to greater knowledge and new translational approaches in pancreatic cancer. These models have seen continuous improvement from the refinement of existing approaches, the meticulous combination of different strains into ever-more complicated combinations, and through the engineering of creative new technologies. Nevertheless, current murine pancreatic cancer models have some shortcomings. For example, although desmoplastic stroma arises in some GEM models of PDAC, it never develops to the extreme levels seen in some human pancreatic tumors. This may just be a function of kinetics since mouse tumors have a few weeks or months to develop while human tumors have years. Nonetheless, this reflects a difference that may have significant impact on the biology and response to therapy of these models.

As with all mouse models of cancer, tumor kinetics remains an issue. In order to be useful for scientific study, one requires a model that develops cancer reasonably quickly. To achieve this, the system is equipped with a large number of mutations in a significant number of cells. It is unclear how much is lost to this drive for "usability", but evidence from numerous models suggests that the tumors that develop most quickly often bear the poorest resemblance to the human disease. Thus, tumors with homozygous deletion/ mutation of $\mathrm{p} 53$ or cdkn2a in addition to Kras ${ }^{\mathrm{G} 12 \mathrm{D}}$ largely develop pancreatic tumors with a sarcomatoid or poorly differentiated histopathology. How to finely balance the competing needs for usability and fidelity will be a constant concern, particularly in the setting of preclinical therapeutics studies.

Another difference pertains to the order of acquisition of genetic alterations during tumor development. While human pancreatic cancer is thought to arise through a stepwise progression beginning with the activation of oncogenes followed by inactivation of tumor suppressors, current mouse models combine both genetic insults at the same time. The use of alternative recombinase systems (such as Flp/ $/ \mathrm{rrt}^{73}$ or Dre/rox ${ }^{74}$ ) in combination with Cre/ lox will enable the phased induction of successive mutations. Additionally, these complementary recombinase systems could be used to separately target different cell types within the tumor.

Another area of refinement is the design of the Cre strains used to activate targeted alleles in the pancreas. Most current models use embryonic pancreatic promoters to drive Cre expression; the mutation of the entire pancreatic anlage might cause a significant field effect throughout the pancreas. A related issue arises with all alleles that harbor a LSL cassette, including Kras ${ }^{\mathrm{LSL} . G 12 \mathrm{D}}$ and $\mathrm{p} 53^{\mathrm{R} 172 \mathrm{H}}$. Because these alleles are phenotypically null prior to recombination, all of the non-recombined cells in the animal are heterozygous knockouts for these alleles. Thus in the KPC model, all of the stromal cells are phenotypically K-ras ${ }^{+/-}$; $\mathrm{p} 53^{+/-}$. There has been no careful investigation reported on the potential for non-cell autonomous haplo insufficiency to impact tumor development or pathology. Ideally, models that switch from wild-type to point-mutant upon activation will be engineered in the future.

There is a continued need for new alleles that will foster our ability to study current models. An imaging reporter strain that can identify the presence of overt carcinomas in a mouse (without marking PanINs) would be invaluable for nearly all types of basic and preclinical studies. Models with biochemically tagged alleles of relevant proteins (such as K-ras) knocked into the endogenous locus would revolutionize the in vivo study of protein biochemistry. Certain molecular systems such as glycosylation pathways, drug metabolisms, and immunology, diverge significantly between human and mouse; the engineering of 
humanized versions of these systems might improve the predictive utility of GEMMs for preclinical studies in addition to providing new basic insights into PDAC biology.

Finally, rapid systems for evaluating candidate gene function in vivo remain a significant challenge. The current approach of crossing individually targeted strains into existing multiallele models is tedious, expensive, and ultimately limiting in its capacity. One new approach was developed by Dieter Sauer and colleagues, who made use of RCAS-mediated retroviral gene transfer to knock down or overexpress target genes in cells that express the retroviral receptor TVA. ${ }^{75,76}$ By restricting the expression of TVA with a LSL cassette, retroviral infection can be limited to those cells that express Cre. This system can therefore be used to target the neoplastic cells of many of the GEMM systems described above. This and other new approaches, such as the "speedy mouse" system developed by Scott Lowe and colleagues, ${ }^{77}$ have yielded significant time savings from optimization of the process, but ultimately entirely new systems for manipulating gene expression in vivo will be desirable to further expedite the study of gene function in vivo. Given the dramatic strides taken in the past decade of development of GEM models of ductal pancreatic cancer, we are excited to see where these new approaches (and others not yet conceived) will lead us in the coming decade.

\section{Non-standard abbreviations}
LSL
loxP-STOP-loxP
PDAC
pancreatic ductal adenocarcinoma
PanIN
pancreatic intraepithelial neoplasia
GEMM
genetically engineered mouse model
GEM
genetically engineered mouse

\section{References}

1. Hidalgo M. Pancreatic cancer. N Engl J Med. 2010; 362:1605-17. [PubMed: 20427809]

2. Vincent A, Herman J, Schulick R, et al. Pancreatic cancer. Lancet. 2011

3. Jones S, Zhang X, Parsons DW, et al. Core signaling pathways in human pancreatic cancers revealed by global genomic analyses. Science. 2008; 321:1801-6. [PubMed: 18772397]

4. Maitra A, Adsay NV, Argani P, et al. Multicomponent analysis of the pancreatic adenocarcinoma progression model using a pancreatic intraepithelial neoplasia tissue microarray. Mod Pathol. 2003; 16:902-12. [PubMed: 13679454]

5. Neesse A, Michl P, Frese KK, et al. Stromal biology and therapy in pancreatic cancer. Gut. 2010

6. Perez-Mancera PA, Guerra C, Barbacid M, et al. What we have learned about pancreatic cancer from mouse models. Gastroenterology. 2012; 142:1079-92. [PubMed: 22406637]

7. Corbett TH, Roberts BJ, Leopold WR, et al. Induction and chemotherapeutic response of two transplantable ductal adenocarcinomas of the pancreas in C57BL/6 mice. Cancer Res. 1984; 44:717-26. [PubMed: 6692374]

8. Longnecker DS. Lesions induced in rodent pancreas by azaserine and other pancreatic carcinogens. Environ Health Perspect. 1984; 56:245-51. [PubMed: 6332732]

9. Jackson EL, Willis N, Mercer K, et al. Analysis of lung tumor initiation and progression using conditional expression of oncogenic K-ras. Genes \& Development. 2001; 15:3243-8. [PubMed: 11751630]

10. Johnson L, Mercer K, Greenbaum D, et al. Somatic activation of the K-ras oncogene causes early onset lung cancer in mice. Nature. 2001; 410:1111-6. [PubMed: 11323676]

11. McGrath JP, Capon DJ, Goeddel DV, et al. Comparative biochemical properties of normal and activated human ras p21 protein. Nature. 1984; 310:644-9. [PubMed: 6147754] 
12. Sweet RW, Yokoyama S, Kamata T, et al. The product of ras is a GTPase and the T24 oncogenic mutant is deficient in this activity. Nature. 1984; 311:273-5. [PubMed: 6148703]

13. Gibbs JB, Sigal IS, Poe M, et al. Intrinsic GTPase activity distinguishes normal and oncogenic ras p21 molecules. Proceedings of the National Academy of Sciences of the United States of America. 1984; 81:5704-8. [PubMed: 6148751]

14. Der CJ, Finkel T, Cooper GM. Biological and biochemical properties of human rasH genes mutated at codon 61. Cell. 1986; 44:167-76. [PubMed: 3510078]

15. Hingorani SR, Jacobetz MA, Robertson GP, et al. Suppression of BRAF(V599E) in human melanoma abrogates transformation. Cancer Res. 2003; 63:5198-202. [PubMed: 14500344]

16. Aguirre AJ, Bardeesy N, Sinha M, et al. Activated Kras and Ink4a/Arf deficiency cooperate to produce metastatic pancreatic ductal adenocarcinoma. Genes Dev. 2003; 17:3112-26. [PubMed: 14681207]

17. Pan FC, Wright C. Pancreas organogenesis: From bud to plexus to gland. Dev Dyn. 2011; 240:530-65. [PubMed: 21337462]

18. Hingorani SR, Petricoin EF, Maitra A, et al. Preinvasive and invasive ductal pancreatic cancer and its early detection in the mouse. Cancer Cell. 2003; 4:437-50. [PubMed: 14706336]

19. Gu G, Dubauskaite J, Melton DA. Direct evidence for the pancreatic lineage: NGN3+ cells are islet progenitors and are distinct from duct progenitors. Development. 2002; 129:2447-57. [PubMed: 11973276]

20. Kawaguchi Y, Cooper B, Gannon M, et al. The role of the transcriptional regulator Ptf1a in converting intestinal to pancreatic progenitors. Nature genetics. 2002; 32:128-34. [PubMed: 12185368]

21. Hingorani SR, Petricoin EF, Maitra A, et al. Preinvasive and invasive ductal pancreatic cancer and its early detection in the mouse. Cancer Cell. 2003; 4:437-50. [PubMed: 14706336]

22. Hingorani SR, Wang L, Multani AS, et al. Trp53R172H and KrasG12D cooperate to promote chromosomal instability and widely metastatic pancreatic ductal adenocarcinoma in mice. Cancer Cell. 2005; 7:469-83. [PubMed: 15894267]

23. Bardeesy N, Cheng K-h, Berger JH, et al. Smad4 is dispensable for normal pancreas development yet critical in progression and tumor biology of pancreas cancer. Genes Dev. 2006; 20:3130-46. [PubMed: 17114584]

24. Morton JP, Timpson P, Karim SA, et al. Mutant p53 drives metastasis and overcomes growth arrest/senescence in pancreatic cancer. Proc Natl Acad Sci U S A. 2010; 107:246-51. [PubMed: 20018721]

25. Muller PA, Caswell PT, Doyle B, et al. Mutant p53 drives invasion by promoting integrin recycling. Cell. 2009; 139:1327-41. [PubMed: 20064378]

26. Ijichi H, Chytil A, Gorska AE, et al. Aggressive pancreatic ductal adenocarcinoma in mice caused by pancreas-specific blockade of transforming growth factor-beta signaling in cooperation with active Kras expression. Genes Dev. 2006; 20:3147-60. [PubMed: 17114585]

27. Bardeesy N, Cheng KH, Berger JH, et al. Smad4 is dispensable for normal pancreas development yet critical in progression and tumor biology of pancreas cancer. Genes Dev. 2006; 20:3130-46. [PubMed: 17114584]

28. Izeradjene K, Combs C, Best M, et al. Kras(G12D) and Smad4/Dpc4 Haploinsufficiency Cooperate to Induce Mucinous Cystic Neoplasms and Invasive Adenocarcinoma of the Pancreas. Cancer Cell. 2007; 11:229-43. [PubMed: 17349581]

29. Collisson EA, Sadanandam A, Olson P, et al. Subtypes of pancreatic ductal adenocarcinoma and their differing responses to therapy. Nat Med. 2011

30. Ying H, Kimmelman AC, Lyssiotis CA, et al. Oncogenic Kras maintains pancreatic tumors through regulation of anabolic glucose metabolism. Cell. 2012; 149:656-70. [PubMed: 22541435]

31. Collins MA, Bednar F, Zhang Y, et al. Oncogenic Kras is required for both the initiation and maintenance of pancreatic cancer in mice. J. Clin. Invest. 2012:1-25.

32. Baines AT, Xu D, Der CJ. Inhibition of Ras for cancer treatment: the search continues. Future medicinal chemistry. 2011; 3:1787-808. [PubMed: 22004085]

33. Stanger BZ, Stiles B, Lauwers GY, et al. Pten constrains centroacinar cell expansion and malignant transformation in the pancreas. Cancer Cell. 2005; 8:185-95. [PubMed: 16169464] 
34. Ying H, Elpek KG, Vinjamoori A, et al. Pten is a major tumor suppressor in pancreatic ductal adenocarcinoma and regulates an NF-кB-cytokine network. Cancer Discovery. 2011; 1:158-169. [PubMed: 21984975]

35. Hill R, Calvopina JH, Kim C, et al. PTEN Loss Accelerates KrasG12D-Induced Pancreatic Cancer Development. Cancer Research. 2010

36. Kojima K, Vickers SM, Adsay NV, et al. Inactivation of Smad4 accelerates Kras(G12D)-mediated pancreatic neoplasia. Cancer Research. 2007; 67:8121-30. [PubMed: 17804724]

37. Heid I, Lubeseder-Martellato C, Sipos B, et al. Early requirement of Rac1 in a mouse model of pancreatic cancer. Gastroenterology. 2011; 141:719-30. 730, e1-7. [PubMed: 21684285]

38. Mann KM, Ward JM, Yew CC, et al. Sleeping Beauty mutagenesis reveals cooperating mutations and pathways in pancreatic adenocarcinoma. Proceedings of the National Academy of Sciences of the United States of America. 2012; 109:5934-41. [PubMed: 22421440]

39. Perez-Mancera PA, Rust AG, van der Weyden L, et al. The deubiquitinase USP9X suppresses pancreatic ductal adenocarcinoma. Nature. 2012; 486:266-70. [PubMed: 22699621]

40. McGrail M, Hatler JM, Kuang X, et al. Somatic mutagenesis with a Sleeping Beauty transposon system leads to solid tumor formation in zebrafish. PLoS One. 2011; 6:e18826. [PubMed: 21533036]

41. Kong B, Michalski CW, Erkan M, et al. From tissue turnover to the cell of origin for pancreatic cancer. Nature reviews Gastroenterology \& hepatology. 2011

42. Kopinke LMD. Pancreatic Stem Cells. StemBook. 2008:1-16.

43. Ku HT. Minireview: pancreatic progenitor cells--recent studies. Endocrinology. 2008; 149:4312-6. [PubMed: 18535096]

44. Gidekel Friedlander SY, Chu GC, Snyder EL, et al. Context-dependent transformation of adult pancreatic cells by oncogenic K-Ras. Cancer Cell. 2009; 16:379-89. [PubMed: 19878870]

45. Morris JP, Cano DA, Sekine S, et al. Beta-catenin blocks Kras-dependent reprogramming of acini into pancreatic cancer precursor lesions in mice. J Clin Invest. 2010; 120:508-20. [PubMed: 20071774]

46. Carrière C, Young AL, Gunn JR, et al. Acute pancreatitis accelerates initiation and progression to pancreatic cancer in mice expressing oncogenic kras in the nestin cell lineage. PLoS ONE. 2011; 6:e27725. [PubMed: 22140463]

47. Carrière C, Seeley ES, Goetze T, et al. The Nestin progenitor lineage is the compartment of origin for pancreatic intraepithelial neoplasia. Proc Natl Acad Sci USA. 2007; 104:4437-42. [PubMed: 17360542]

48. Kopp JL, Dubois CL, Schaffer AE, et al. Sox9+ ductal cells are multipotent progenitors throughout development but do not produce new endocrine cells in the normal or injured adult pancreas. Development. 2011; 138:653-65. [PubMed: 21266405]

49. Furuyama K, Kawaguchi Y, Akiyama H, et al. Continuous cell supply from a Sox9-expressing progenitor zone in adult liver, exocrine pancreas and intestine. Nat Genet. 2010

50. Stanger BZ, Dor Y. Dissecting the cellular origins of pancreatic cancer. Cell Cycle. 2006; 5:43-6. [PubMed: 16322695]

51. Kopinke D, Brailsford M, Pan FC, et al. Ongoing Notch signaling maintains phenotypic fidelity in the adult exocrine pancreas. Developmental Biology. 2012; 362:57-64. [PubMed: 22146645]

52. Bao B, Wang Z, Ali S, et al. Notch-1 induces epithelial-mesenchymal transition consistent with cancer stem cell phenotype in pancreatic cancer cells. CANCER LETTERS. 2011

53. Quante M, Wang TC. Stem cells in gastroenterology and hepatology. Nat Rev Gastroenterol Hepatol. 2009; 6:724-37. [PubMed: 19884893]

54. Clark CE, Hingorani SR, Mick R, et al. Dynamics of the immune reaction to pancreatic cancer from inception to invasion. Cancer Res. 2007; 67:9518-27. [PubMed: 17909062]

55. Pylayeva-Gupta Y, Lee KE, Hajdu CH, et al. Oncogenic Kras-Induced GM-CSF Production Promotes the Development of Pancreatic Neoplasia. Cancer Cell. 2012; 21:836-847. [PubMed: 22698407] 
56. Bayne LJ, Beatty GL, Jhala N, et al. Tumor-derived granulocyte-macrophage colony-stimulating factor regulates myeloid inflammation and $\mathrm{T}$ cell immunity in pancreatic cancer. Cancer Cell. 2012; 21:822-35. [PubMed: 22698406]

57. Olive KP, Jacobetz MA, Davidson CJ, et al. Inhibition of Hedgehog signaling enhances delivery of chemotherapy in a mouse model of pancreatic cancer. Science. 2009; 324:1457-61. [PubMed: 19460966]

58. Thayer SP, di Magliano MP, Heiser PW, et al. Hedgehog is an early and late mediator of pancreatic cancer tumorigenesis. Nature. 2003; 425:851-6. [PubMed: 14520413]

59. Tian H, Callahan CA, DuPree KJ, et al. Hedgehog signaling is restricted to the stromal compartment during pancreatic carcinogenesis. Proc Natl Acad Sci U S A. 2009; 106:4254-9. [PubMed: 19246386]

60. Nolan-Stevaux O, Lau J, Truitt ML, et al. GLI1 is regulated through Smoothened-independent mechanisms in neoplastic pancreatic ducts and mediates PDAC cell survival and transformation. Genes Dev. 2009; 23:24-36. [PubMed: 19136624]

61. Bailey JM, Mohr AM, Hollingsworth MA. Sonic hedgehog paracrine signaling regulates metastasis and lymphangiogenesis in pancreatic cancer. Oncogene. 2009; 28:3513-25. [PubMed: 19633682]

62. Bailey JM, Swanson BJ, Hamada T, et al. Sonic hedgehog promotes desmoplasia in pancreatic cancer. Clin Cancer Res. 2008; 14:5995-6004. [PubMed: 18829478]

63. Apelqvist A, Li H, Sommer L, et al. Notch signalling controls pancreatic cell differentiation. Nature. 1999; 400:877-81. [PubMed: 10476967]

64. Miyamoto Y, Maitra A, Ghosh B, et al. Notch mediates TGF alpha-induced changes in epithelial differentiation during pancreatic tumorigenesis. Cancer Cell. 2003; 3:565-76. [PubMed: 12842085]

65. Ranganathan P, Weaver KL, Capobianco AJ. Notch signalling in solid tumours: a little bit of everything but not all the time. Nature Publishing Group. 2011; 11:336-351.

66. Cook N, Olive KP, Frese K, et al. K-ras-driven pancreatic cancer mouse model for anticancer inhibitor analyses. Methods Enzymol. 2008; 439:73-85. [PubMed: 18374157]

67. Singh M, Lima A, Molina R, et al. Assessing therapeutic responses in Kras mutant cancers using genetically engineered mouse models. Nat Biotechnol. 2010; 28:585-93. [PubMed: 20495549]

68. Jacobetz MA, Chan DS, Neesse A, et al. Hyaluronan impairs vascular function and drug delivery in a mouse model of pancreatic cancer. Gut. 2012

69. Provenzano PP, Cuevas C, Chang AE, et al. Enzymatic targeting of the stroma ablates physical barriers to treatment of pancreatic ductal adenocarcinoma. Cancer Cell. 2012; 21:418-29. [PubMed: 22439937]

70. Frese KK, Neesse A, Cook N, et al. nab-Paclitaxel Potentiates Gemcitabine Activity by Reducing Cytidine Deaminase Levels in a Mouse Model of Pancreatic Cancer. Cancer discovery. 2012; 2:260-9. [PubMed: 22585996]

71. Von Hoff DD, Ramanathan RK, Borad MJ, et al. Gemcitabine Plus nab-Paclitaxel Is an Active Regimen in Patients With Advanced Pancreatic Cancer: A Phase I/II Trial. Journal of Clinical Oncology. 2011

72. Beatty GL, Chiorean EG, Fishman MP, et al. CD40 agonists alter tumor stroma and show efficacy against pancreatic carcinoma in mice and humans. Science. 2011; 331:1612-6. [PubMed: 21436454]

73. Gama, Sosa MA.; De Gasperi, R.; Elder, GA. Animal transgenesis: an overview. Brain structure \& function. 2010; 214:91-109. [PubMed: 19937345]

74. Anastassiadis K, Fu J, Patsch C, et al. Dre recombinase, like Cre, is a highly efficient site-specific recombinase in E. coli, mammalian cells and mice. Disease models \& mechanisms. 2009; 2:50815. [PubMed: 19692579]

75. Mayr U, von Werder A, Seidler B, et al. RCAS-mediated retroviral gene delivery: a versatile tool for the study of gene function in a mouse model of pancreatic cancer. Hum Gene Ther. 2008; 19:896-906. [PubMed: 18759559] 
76. Seidler B, Schmidt A, Mayr U, et al. A Cre-loxP-based mouse model for conditional somatic gene expression and knockdown in vivo by using avian retroviral vectors. Proc Natl Acad Sci U S A. 2008; 105:10137-42. [PubMed: 18621715]

77. Heyer J, Kwong LN, Lowe SW, et al. Non-germline genetically engineered mouse models for translational cancer research. Nature reviews. Cancer. 2010; 10:470-80.

78. De La OJ-P, Emerson LL, Goodman JL, et al. Notch and Kras reprogram pancreatic acinar cells to ductal intraepithelial neoplasia. Proc Natl Acad Sci USA. 2008; 105:18907-12. [PubMed: 19028876]

79. Wagner M, Greten FR, Weber CK, et al. A murine tumor progression model for pancreatic cancer recapitulating the genetic alterations of the human disease. Genes \& Development. 2001; 15:28693. [PubMed: 11159909]

80. Guerra C, Schuhmacher AJ, Cañamero M, et al. Chronic pancreatitis is essential for induction of pancreatic ductal adenocarcinoma by K-Ras oncogenes in adult mice. Cancer Cell. 2007; 11:291302. [PubMed: 17349585]

81. Morton JP, Jamieson NB, Karim SA, et al. LKB1 haploinsufficiency cooperates with Kras to promote pancreatic cancer through suppression of p21-dependent growth arrest. Gastroenterology. 2010; 139:586-97. 597, e1-6. [PubMed: 20452353]

82. Skoulidis F, Cassidy LD, Pisupati V, et al. Germline Brca2 Heterozygosity Promotes Kras(G12D) -Driven Carcinogenesis in a Murine Model of Familial Pancreatic Cancer. Cancer Cell. 2010

83. Bardeesy N, Aguirre AJ, Chu GC, et al. Both p16(Ink4a) and the p19(Arf)-p53 pathway constrain progression of pancreatic adenocarcinoma in the mouse. Proc Natl Acad Sci USA. 2006; 103:5947-52. [PubMed: 16585505]

84. Shakya R, Reid LJ, Reczek CR, et al. BRCA1 tumor suppression depends on BRCT phosphoprotein binding, but not its E3 ligase activity. Science. 2011; 334:525-8. [PubMed: 22034435]

85. Rowley M, Ohashi A, Mondal G, et al. Inactivation of Brca2 promotes Trp53-associated but inhibits KrasG12D-dependent pancreatic cancer development in mice. Gastroenterology. 2011; 140:1303-1313. e1-3. [PubMed: 21199651]

86. Carrière C, Gore AJ, Norris AM, et al. Deletion of Rb Accelerates Pancreatic Carcinogenesis by Oncogenic Kras and Impairs Senescence in Premalignant Lesions. Gastroenterology. 2011

87. Hezel AF, Gurumurthy S, Granot Z, et al. Pancreatic LKB1 deletion leads to acinar polarity defects and cystic neoplasms. Molecular and cellular biology. 2008; 28:2414-25. [PubMed: 18227155]

88. Heiser PW, Cano DA, Landsman L, et al. Stabilization of beta-catenin induces pancreas tumor formation. Gastroenterology. 2008; 135:1288-300. [PubMed: 18725219]

89. Maniati E, Bossard M, Cook N, et al. Crosstalk between the canonical NF- $\ltimes$ B and Notch signaling pathways inhibits Ppary expression and promotes pancreatic cancer progression in mice. The Journal of clinical investigation. 2011

90. Siveke JT, Einwachter H, Sipos B, et al. Concomitant pancreatic activation of Kras(G12D) and Tgfa results in cystic papillary neoplasms reminiscent of human IPMN. Cancer Cell. 2007; 12:266-79. [PubMed: 17785207]

91. Tinder TL, Subramani DB, Basu GD, et al. MUC1 enhances tumor progression and contributes toward immunosuppression in a mouse model of spontaneous pancreatic adenocarcinoma. Journal of immunology. 2008; 181:3116-25.

92. Mazur PK, Einwachter H, Lee M, et al. Notch2 is required for progression of pancreatic intraepithelial neoplasia and development of pancreatic ductal adenocarcinoma. Proceedings of the National Academy of Sciences of the United States of America. 2010; 107:13438-43. [PubMed: 20624967]

93. Nolan-Stevaux O, Lau J, Truitt ML, et al. GLI1 is regulated through Smoothened-independent mechanisms in neoplastic pancreatic ducts and mediates PDAC cell survival and transformation. Genes \& Development. 2009; 23:24-36. [PubMed: 19136624]

94. Ijichi H, Chytil A, Gorska AE, et al. Inhibiting Cxcr2 disrupts tumor-stromal interactions and improves survival in a mouse model of pancreatic ductal adenocarcinoma. The Journal of clinical investigation. 2011 
95. Feldmann G, Fendrich V, McGovern K, et al. An orally bioavailable small-molecule inhibitor of Hedgehog signaling inhibits tumor initiation and metastasis in pancreatic cancer. Molecular Cancer Therapeutics. 2008; 7:2725-35. [PubMed: 18790753]

96. Olive KP, Jacobetz MA, Davidson CJ, et al. Inhibition of Hedgehog signaling enhances delivery of chemotherapy in a mouse model of pancreatic cancer. Science. 2009; 324:1457-61. [PubMed: 19460966]

97. Solan JL, Hingorani SR, Lampe PD. Changes in connexin43 expression and localization during pancreatic cancer progression. The Journal of membrane biology. 2012; 245:255-62. [PubMed: 22729649]

98. Cook N, Frese KK, Bapiro TE, et al. Gamma secretase inhibition promotes hypoxic necrosis in mouse pancreatic ductal adenocarcinoma. The Journal of experimental medicine. 2012 


\begin{tabular}{|c|c|c|c|c|c|c|c|c|c|c|c|c|c|c|c|c|c|c|c|c|c|}
\hline है & 葽 & & 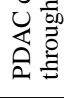 & & $\begin{array}{l}2 \\
0 \\
0 \\
n\end{array}$ & 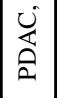 & 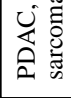 & 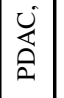 & 嵀 & 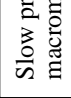 & 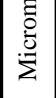 & 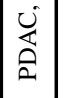 & 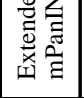 & 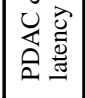 & 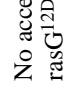 & 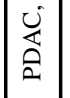 & & 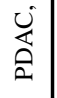 & 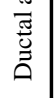 & $\begin{array}{l}\overrightarrow{0} \\
\overline{0} \\
3 \\
3 \\
3\end{array}$ & 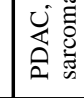 \\
\hline $\begin{array}{l}\bar{\pi} \\
\bar{n} \\
\bar{n}\end{array}$ & 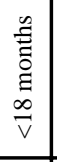 & 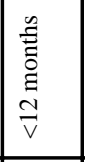 & 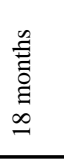 & 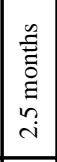 & 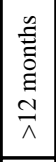 & 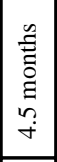 & $\frac{\dot{I}}{\mathrm{Z}}$ & 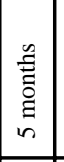 & 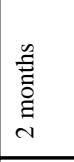 & $\begin{array}{l}\text { 㪯 } \\
\text { ! } \\
\underline{\Xi}\end{array}$ & 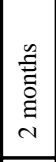 & 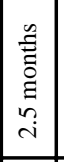 & $\overleftrightarrow{\mathrm{z}}$ & 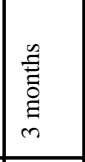 & $\overleftrightarrow{\mathrm{z}}$ & 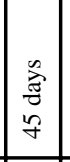 & 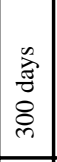 & 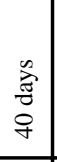 & 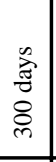 & 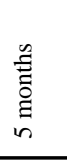 & 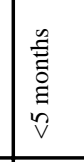 \\
\hline 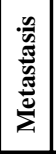 & $\stackrel{\circ}{z}$ & $\stackrel{z}{z}$ & $\stackrel{8}{z}$ & $\stackrel{\circ}{z}$ & 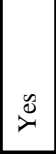 & $\mathbb{Z}$ & $\overleftrightarrow{\mathrm{z}}$ & $\stackrel{0}{\infty}$ & $\stackrel{\tilde{c}}{\stackrel{0}{0}}$ & $\check{\nu}^{\infty}$ & $\widehat{\widehat{B}}$ & $\overleftrightarrow{\mathrm{z}}$ & z & $\stackrel{\circ}{z}$ & $\overleftrightarrow{z}$ & $\overleftrightarrow{z}$ & $\overleftrightarrow{\mathrm{z}}$ & $\overleftrightarrow{\Sigma}$ & $\overleftrightarrow{z}$ & $\stackrel{\infty}{\check{\infty}}$ & $\overleftrightarrow{\underline{z}}$ \\
\hline 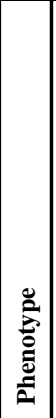 & $\begin{array}{l}\text { za } \\
\text { ज्ञ } \\
\text { है }\end{array}$ & 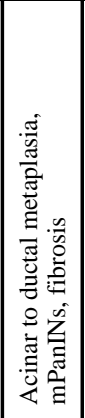 & 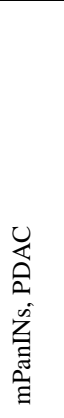 & 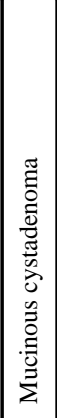 & 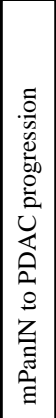 & 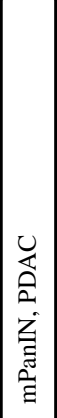 & 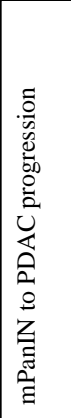 & 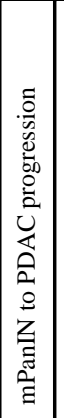 & 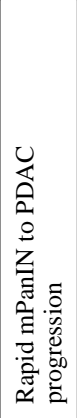 & 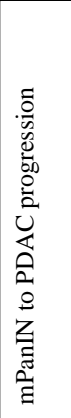 & 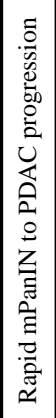 & 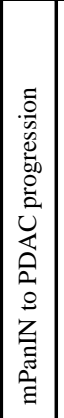 & 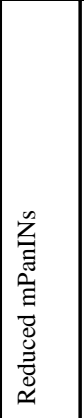 & 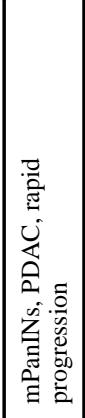 & 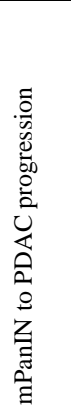 & 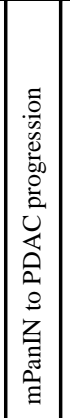 & 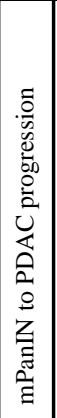 & 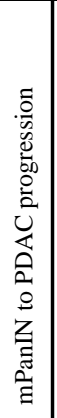 & 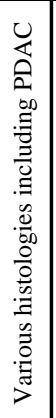 & 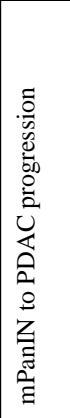 & 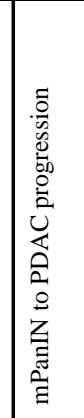 \\
\hline$\frac{\frac{\tilde{g}}{\varrho}}{=}$ & 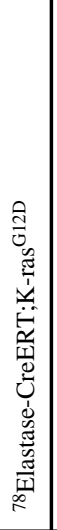 & 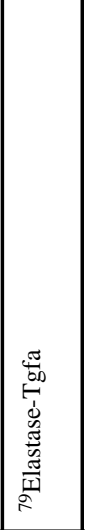 & 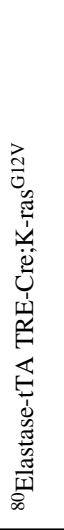 & 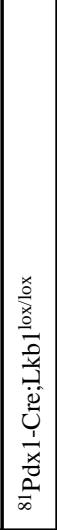 & 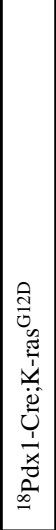 & 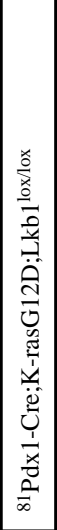 & 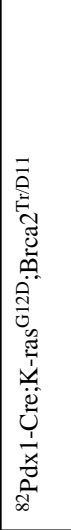 & 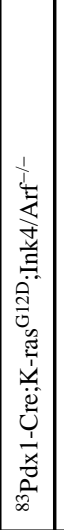 & 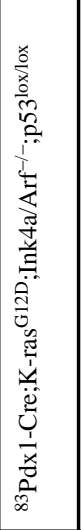 & 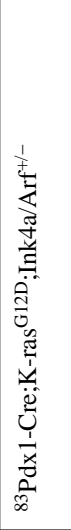 & 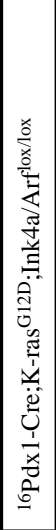 & 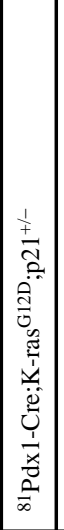 & 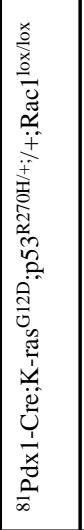 & 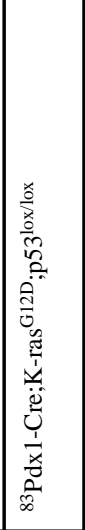 & 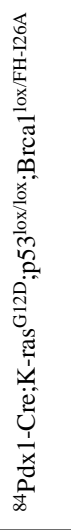 & 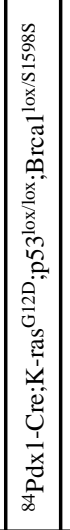 & 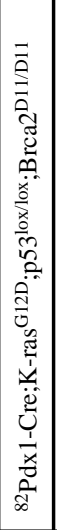 & 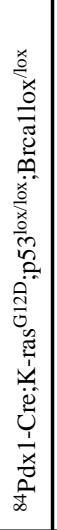 & 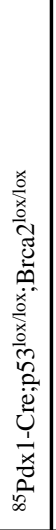 & 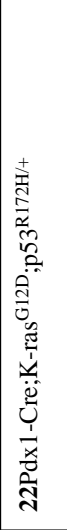 & 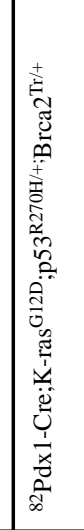 \\
\hline
\end{tabular}




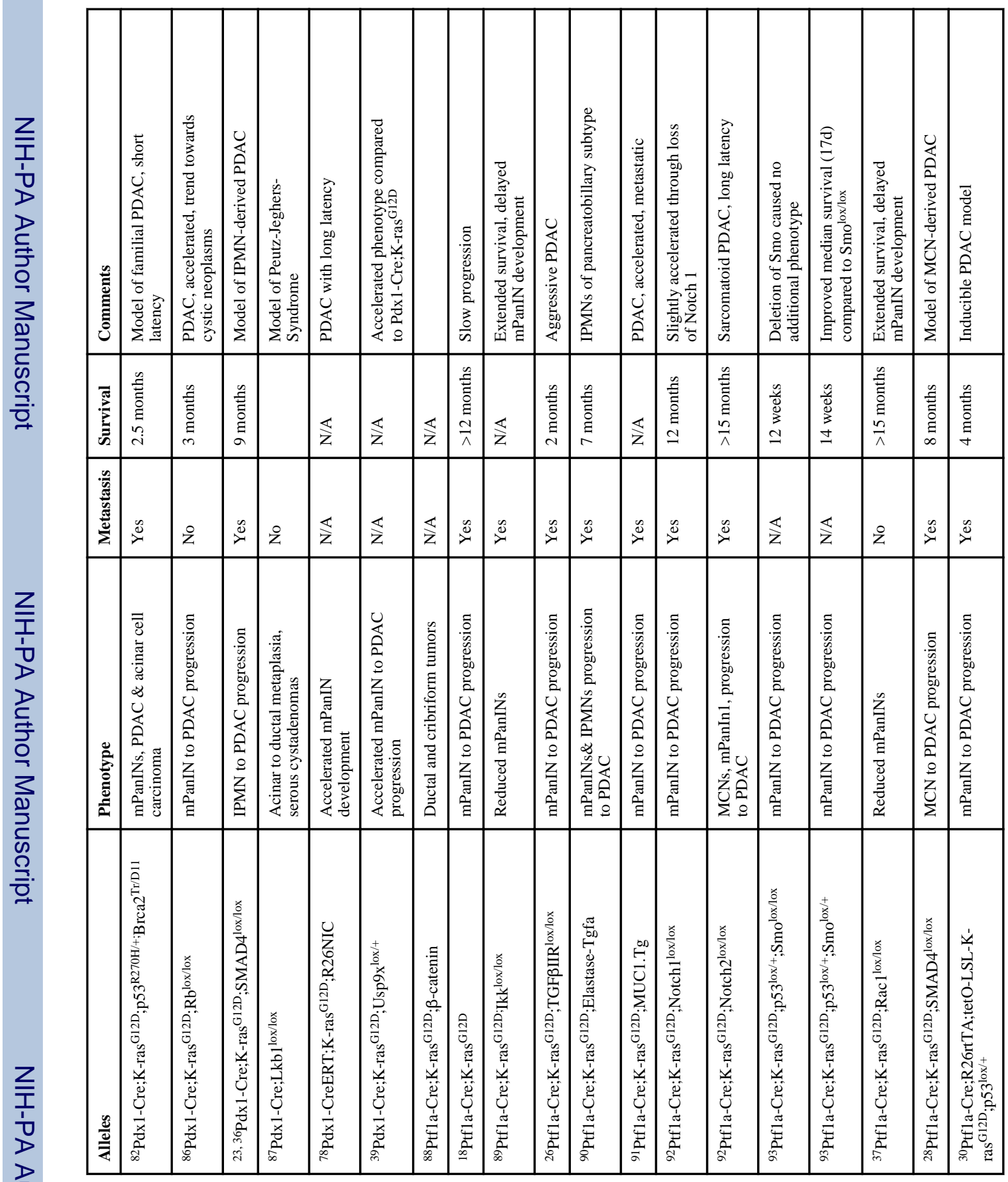




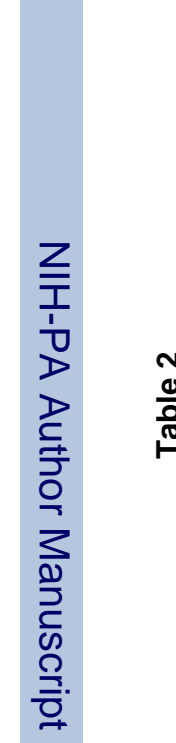

\begin{tabular}{|c|c|c|c|c|c|c|c|c|c|c|c|c|}
\hline & 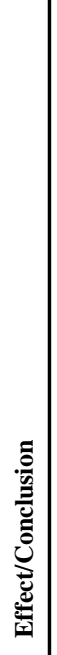 & 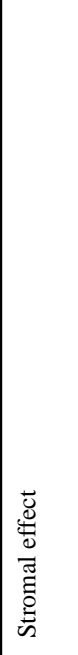 & 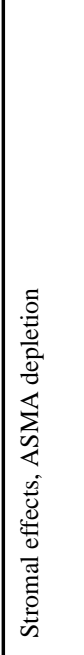 & 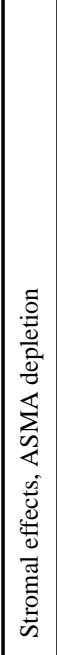 & 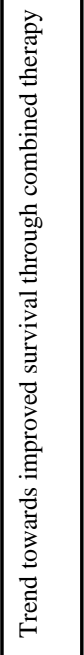 & 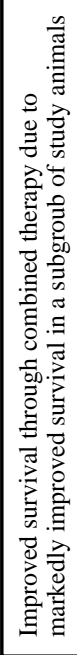 & 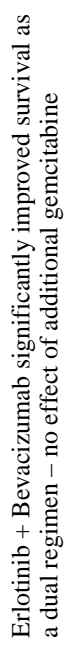 & 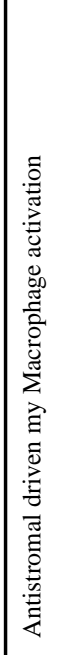 & 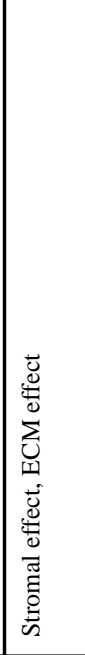 & 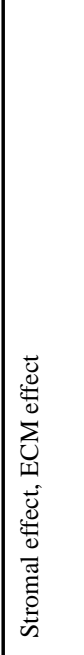 & 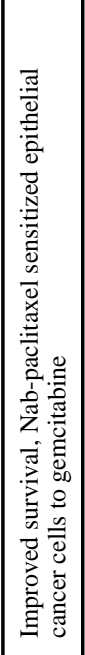 & 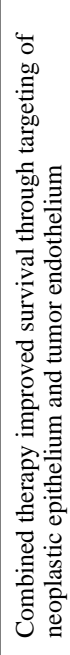 \\
\hline 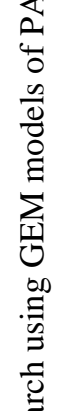 & 悹 & 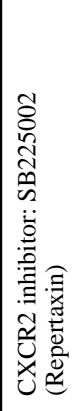 & 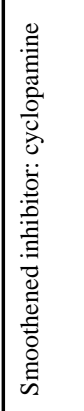 & 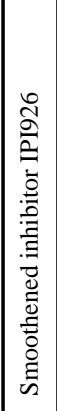 & 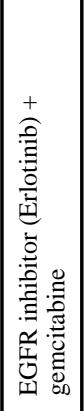 & 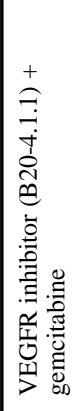 & 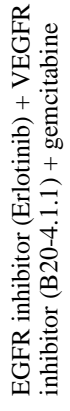 & 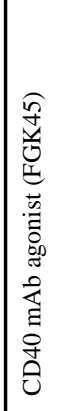 & 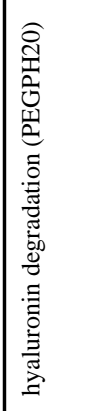 & 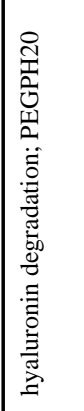 & 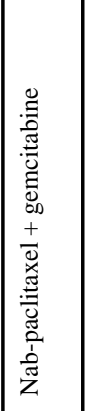 & 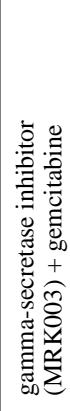 \\
\hline$\frac{\tilde{s}}{2}$ & 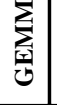 & & & 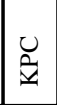 & & & & $\frac{U}{\grave{z}}$ & $\frac{U}{\grave{z}}$ & 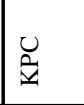 & 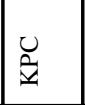 & $\frac{\partial}{\Delta}$ \\
\hline 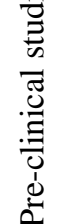 & 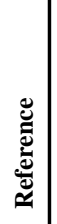 & 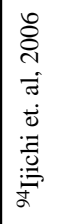 & 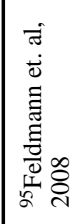 & 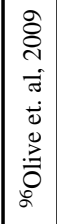 & 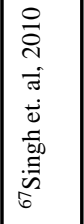 & 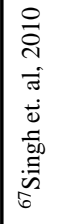 & 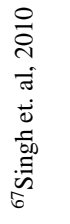 & 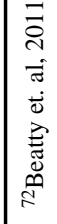 & 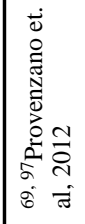 & 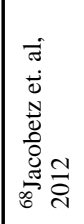 & 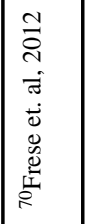 & 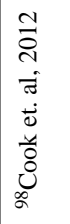 \\
\hline
\end{tabular}

Cancer J. Author manuscript; available in PMC 2013 November 01. 